\title{
On Robust Stability of Discrete-Time Adaptive Nonlinear Control *广
}

\author{
Chanying Li and Liang-Liang Xie \\ Institute of Systems Science \\ Chinese Academy of Sciences \\ Beijing, 100080, China
}

Submitted, May 3, 2004; Revised, May 30, 2005; September 12, 2005

\begin{abstract}
We consider adaptive control of discrete-time nonlinear systems with a single unknown parameter in this paper. We demonstrate that the necessary and sufficient condition for the existence of a feedback stabilizer that is robust to bounded noise is that the nonlinear growth rate of the system dynamics is less than 4 . This result further confirms the conclusion of [1] which addresses unbounded noise in a stochastic setting. Also in our worst-case approach, we find that much simpler adaptive stabilizers can be constructed when the nonlinear growth rate is less than 4 .
\end{abstract}

Keywords: Adaptive control; Nonlinear systems; Robust stability; Discrete-time systems; Feedback capability

\footnotetext{
*Please address all correspondence to the second author Prof. L.-L. Xie. (xie@control.iss.ac.cn)

${ }^{\dagger}$ Partially supported by the National Natural Science Foundation of China and the National Key Project of China.
} 


\section{Introduction}

On nonlinear adaptive control, much less results are available in the literature for discretetime systems, compared with continuous-time systems. The difficulty involved with adaptive control of discrete-time nonlinear systems was clearly demonstrated by the negative conclusion drawn in [1], which states that it is impossible in general to stabilize a discretetime nonlinear system with even only one unknown parameter if the nonlinear growth rate is too high. In contrast, for a continuous-time counter-part, no matter how high the nonlinear growth rate is, it can always be stabilized by, say, a nonlinear damping controller with a higher order.

The benchmark model considered by [1] is as follows:

$$
y_{t+1}=\theta y_{t}^{b}+u_{t}+w_{t+1}, \quad t=0,1, \ldots
$$

where, $u_{t}, y_{t}$ and $w_{t}$ are the system input, output and noise respectively, $\theta$ is an unknown parameter, and the exponent $b \geq 1$ is a known real number and is regarded as the nonlinear growth rate of the system.

For the system (1), under the assumption that both the unknown parameter $\theta$ and the noise $\left\{w_{t}\right\}$ are Gaussian distributed, [1] proved that if the nonlinear growth rate $b \geq 4$, then for any causal feedback control, there always exists a set with positive probability, on which the closed-loop dynamics is unstable. On the other hand, if $b<4$, it was shown in [1] that the standard least-square-based adaptive control scheme can ensure the closed-loop stability almost surely.

Later on, [4] extended the negative conclusion of [1] to systems with multiple unknown parameters, and proved a polynomial rule, which implies that generally linear growth condition is indispensable for almost sure stability if no constraint is exerted on the number of unknown parameters. [5] further demonstrated that the same polynomial rule holds even if the uncertain parameters are known a priori to lie in a bounded region. 
All these results mentioned above assume Gaussian distributed noise. It would be interesting to ask what happens if the noise are bounded. Are there still negative conclusions that prevent the existence of a stabilizing feedback controller for any nonlinear growth rate? If yes, do they have the same constraints on the nonlinear growth rate?

We still take the model (1) as the starting point to answer these questions. Here in this paper, instead, we assume bounded noise. One may suspect that the boundedness assumption on the noise $w_{t}$ would be helpful for designing feedback stabilizers, which would at least result in a looser requirement on the nonlinear growth rate $b$. In fact, we will demonstrate the contrary. We will show that $b<4$ is still necessary for the existence of a feedback stabilizer, even if the noise are assumed to be uniformly bounded and with the bound known a priori. However, the boundedness assumption on the noise will indeed be helpful in designing much simpler feedback stabilizers when $b<4$.

Other related works include some papers considering noise-free models (see e.g. [2, 3, 7]). But there is a fundamental drawback with such models. The trick lies in that without noise, the parameters are completely solvable with linear equations. For example, without $w_{1}$ in (1), $\theta$ can be completely determined by $y_{1}, y_{0}$ and $u_{0}$ with the equation

$$
y_{1}=\theta y_{0}^{b}+u_{0}
$$

Of course this kind of equation-solving methods are not useful in practice due to that they are not robust to the noise. While all the authors realized this and thereby came up with some other (mostly recursive) types of parameter estimation algorithms, it is always impossible to justify the robustness of those algorithms thus obtained without explicitly considering noise. A good example is the weighted-least-square-based adaptive controller constructed in [3], which was shown capable of stabilizing (1) for any nonlinear growth rate $b \geq 1$, but with all $w_{t}=0$. Interestingly, one implication of the results in this paper is just that such a controller cannot be robust to bounded noise at least for the case $b \geq 4$. 


\section{Main Results}

Consider the system (1) with the following assumptions.

A1) At the time $t=0$, the a priori knowledge about the unknown parameter $\theta$ is that it can be any value on some interval $[\underline{\theta}, \bar{\theta}] \subset \mathbb{R}^{1}$.

A2) The noise are assumed to be bounded with the bound $w>0$, i.e.,

$$
\sup _{t \geq 1}\left|w_{t}\right| \leq w
$$

We are interested in designing a feedback control law which robustly stabilizes the system (1) with respect to any possible $\theta$ and $\left\{w_{t}\right\}$ under the assumptions A1)-A2).

First, we restate the definition of a feedback control law, which has appeared in [6].

Definition 2.1 A sequence $\left\{u_{t}\right\}$ is called a feedback control law if at any time $t \geq 0$, $u_{t}$ is a (causal) function of all the observations up to the time $t:\left\{y_{i}, i \leq t\right\}$, i.e.,

$$
u_{t}=h_{t}\left(y_{0}, \cdots, y_{t}\right)
$$

where $h_{t}(\cdot): \mathbb{R}^{t+1} \rightarrow \mathbb{R}^{1}$ can be any (nonlinear) mapping.

Although there is no unified definition of adaptive control, it is generally thought of as a combination of two parts: online parameter estimation plus controller design with updated parameter estimates. Anyway, it must be causal. That is, whatever the adaptive control law designed, it is one feedback control law in Definition 2.1.

Definition 2.2 The system (1) under the assumptions A1)-A2) is said to be robust feedback stabilizable, if there exists a feedback control law $\left\{u_{t}\right\}$ such that for any $y_{0} \in \mathbb{R}^{1}$ and any $\theta,\left\{w_{t}\right\}$ satisfying A1)-A2), the outputs of the closed-loop system are bounded as follows:

$$
\sup _{t \geq 0}\left|y_{t}\right|<\infty .
$$


Remark 2.1 The stability defined in Definition 2.2 applies to any possible $y_{0} \in \mathbb{R}^{1}$ and any possible realization of $\theta$ and $\left\{w_{t}\right\}$ which satisfy A1)-A2). A robust feedback stabilizer should be able to deal with any possible realization of $y_{0}, \theta$ and $\left\{w_{t}\right\}$. We don't assume any statistical behavior of these unknown parameters. Hence this stability is defined in the sense of the worst case that can happen, although it may only be one specific sample path. This is one key difference between our approach and the stochastic setting in [1]. But the stability in Definition 2.2 is very week in that the bounds (4) need not be the same for all the realizations of $y_{0}, \theta$ and $\left\{w_{t}\right\}$.

Theorem 2.1 The system (1) under the assumptions A1)-A2) is robust feedback stabilizable if and only if $b<4$.

Next, consider a more general model:

$$
y_{t+1}=\theta f\left(y_{t}\right)+u_{t}+w_{t+1}
$$

where $f: \mathbb{R}^{1} \rightarrow \mathbb{R}^{1}$ is a known nonlinear mapping. We consider the following assumptions on $f(\cdot)$.

A3) There exist $a_{1}>0, b>0$ and $M_{1}>0$ such that

$$
\begin{aligned}
& |f(x)| \leq a_{1}|x|^{b}, \quad \text { for }|x| \geq M_{1} \\
& \text { and } \sup _{|x| \leq M_{1}}|f(x)|<\infty .
\end{aligned}
$$

A4) There exist $a_{2}>0, b>0$ and $M_{2}>0$ such that

$$
|f(x)| \geq a_{2}|x|^{b}, \quad \text { for }|x| \geq M_{2}
$$

Intuitively, the assumption A3) exerts an upper bound on the growth rate of the system dynamics; and conversely, the assumption A4) exerts a lower bound on the growth rate of the system dynamics. For either of them, we have one corresponding conclusion as follows. 
Theorem 2.2 The system (5) under the assumptions A1)-A3) is robust feedback stabilizable if $b<4$.

Theorem 2.3 The system (5) under the assumptions A1)-A2) and A4) is not robust feedback stabilizable if $b \geq 4$.

The combination of Theorems 2.2 and 2.3 immediately leads to the following conclusion.

Corollary 2.1 The system (5) under the assumptions A1)-A4) is robust feedback stabilizable if and only if $b<4$.

\section{Proof of the Theorems}

Proof of Theorem 2.1: Sufficiency: We show a simple adaptive control law, which robustly stabilizes the system (1) for any $b<4$. We will also see that to implement this algorithm, the bounds $[\underline{\theta}, \bar{\theta}]$ and $w$ need not to be known.

Without loss of generality, suppose $y_{0} \neq 0$.

For any $t \geq 1$, let

$$
i_{t}:=\underset{0 \leq i \leq t-1}{\operatorname{argmax}}\left|y_{i}\right|
$$

That is,

$$
\left|y_{i_{t}}\right|=\max _{0 \leq i \leq t-1}\left|y_{i}\right|
$$

The parameter estimate at the time $t \geq 1$ is chosen to be

$$
\hat{\theta}_{t}:=\frac{y_{i_{t}+1}-u_{i_{t}}}{y_{i_{t}}^{b}}
$$

It is easy to check by (1) that the estimation error is

$$
\tilde{\theta}_{t}:=\theta-\hat{\theta}_{t}=\frac{-w_{i_{t}+1}}{y_{i_{t}}^{b}}
$$


Now we define the control sequence as the following.

$$
\begin{aligned}
& u_{0}=0, \\
& u_{t}=-\hat{\theta}_{t} y_{t}^{b}, \quad \text { for } t \geq 1 .
\end{aligned}
$$

Then for $t \geq 1$, the closed-loop dynamics is

$$
y_{t+1}=\tilde{\theta}_{t} y_{t}^{b}+w_{t+1}=\frac{-w_{i_{t}+1}}{y_{i_{t}}^{b}} y_{t}^{b}+w_{t+1}
$$

Therefore, noting that the noise are uniformly bounded as (2), we have

$$
\left|y_{t+1}\right| \leq \frac{w}{\left|y_{i_{t}}\right|^{b}}\left|y_{t}\right|^{b}+w, \quad \text { for any } t \geq 1
$$

Then by (10), we have

$$
\left|y_{t+1}\right| \leq \frac{w}{\max _{0 \leq i \leq t-1}\left|y_{i}\right|^{b}}\left|y_{t}\right|^{b}+w, \quad \text { for any } t \geq 1
$$

Now we use a contradiction argument to prove that the outputs are uniformly bounded as (4). Suppose on the contrary, there exist some $y_{0} \in \mathbb{R}^{1}$, and some $\theta$ and a sequence of $\left\{w_{t}\right\}$ satisfying the assumptions A1)-A2) such that for the feedback control law proposed above,

$$
\sup _{t \geq 0}\left|y_{t}\right|=\infty
$$

Then from this sequence $\left\{\left|y_{t}\right|, t \geq 0\right\}$, we can pick out a subsequence $\left\{\left|y_{t_{k}}\right|, k \geq 1\right\}$ which monotonously increasing to the infinity and also for any $k=1,2, \ldots$

$$
\left|y_{t}\right| \leq\left|y_{t_{k}}\right|<\left|y_{t_{k+1}}\right| \quad \text { for any } t_{k}<t<t_{k+1} \text {. }
$$

For any $k=2,3, \ldots$, by (16), we have

$$
\left|y_{t_{k+1}}\right| \leq \frac{w}{\max _{0 \leq i \leq t_{k+1}-2}\left|y_{i}\right|^{b}}\left|y_{t_{k+1}-1}\right|^{b}+w .
$$


By (17), it is easy to check that

$$
\left|y_{t_{k+1}-1}\right| \leq\left|y_{t_{k}}\right| \text { and } \max _{0 \leq i \leq t_{k+1}-2}\left|y_{i}\right| \geq\left|y_{t_{k-1}}\right|
$$

Hence, by (18), we have

$$
\left|y_{t_{k+1}}\right| \leq \frac{w}{\left|y_{t_{k-1}}\right|^{b}}\left|y_{t_{k}}\right|^{b}+w
$$

Take logarithm on both sides of (19), and noting that $\left|y_{t_{k}}\right|>\left|y_{t_{k-1}}\right|$, we have

$$
\begin{aligned}
\log \left|y_{t_{k+1}}\right| & \leq \log \left(\frac{w}{\left|y_{t_{k-1}}\right|^{b}}\left|y_{t_{k}}\right|^{b}+w\right) \\
& <\log \left(2 w \frac{\left|y_{t_{k}}\right|^{b}}{\left|y_{t_{k-1}}\right|^{b}}\right) \\
& =b\left(\log \left|y_{t_{k}}\right|-\log \left|y_{t_{k-1}}\right|\right)+\log (2 w) .
\end{aligned}
$$

Then it follows by Lemma 3.5 in [6] that for $b<4,\left\{\log \left|y_{t_{k}}\right|\right\}$ cannot be monotonously increasing to the infinity, which contradicts to the definition of $\left\{\left|y_{t_{k}}\right|\right\}$. This concludes the contradiction argument.

Necessity: We show that if $b \geq 4$ and $\left|y_{0}\right|$ is sufficiently large, then for any feedback control law, there always exist some $\theta$ and $\left\{w_{t}\right\}$ satisfying the assumptions A1)-A2) such that the absolute values of the outputs monotonously increase to the infinity:

$$
\left|y_{t}\right| \nearrow \infty
$$

First, we introduce some notation. For any interval $A=\left[a_{1}, a_{2}\right] \subset \mathbb{R}^{1}$, let $|A|=a_{2}-a_{1}$. Let $\Theta_{0}:=[\underline{\theta}, \bar{\theta}]$, then $\left|\Theta_{0}\right|=\bar{\theta}-\underline{\theta}$.

Since $b \geq 4$, it is easy to see that $b-2 \sqrt{b} \geq 0$. We consider sufficiently large $\left|y_{0}\right|$ 
satisfying the following inequalities:

$$
\begin{gathered}
\frac{1}{4}\left|\Theta_{0}\right| \cdot\left|y_{0}\right|^{b} \geq 2 w \\
\frac{1}{8}\left|\Theta_{0}\right| \cdot\left|y_{0}\right|^{b-\sqrt{b}} \geq \frac{8}{w} \\
\left(\frac{8}{w}\right)^{b}\left|y_{0}\right|^{b \sqrt{b}-b} \geq 8 \\
\left(\frac{8}{w}\right)^{\sqrt{b}-2}\left|y_{0}\right|^{b-2 \sqrt{b}} \geq 1 .
\end{gathered}
$$

At the time $t=0$, the system dynamics evolves as

$$
y_{1}=\theta y_{0}^{b}+u_{0}+w_{1}
$$

Since $\theta$ can be any value on the interval $\Theta_{0}=[\underline{\theta}, \bar{\theta}], \theta y_{0}^{b}$ can be any value on the interval $\left[\underline{\theta} y_{0}^{b}, \bar{\theta} y_{0}^{b}\right]$, which is of length $\left|\Theta_{0}\right| \cdot\left|y_{0}\right|^{b}$. Then for any fixed choice ${ }^{1}$ of $u_{0} \in \mathbb{R}^{1}$, there always exists some interval

$$
\Theta_{0}^{\prime}=\left[\underline{\theta}_{0}, \bar{\theta}_{0}\right] \subset \Theta_{0}
$$

which depends on $u_{0}$ and is of the length

$$
\left|\Theta_{0}^{\prime}\right| \geq \frac{1}{4}\left|\Theta_{0}\right|
$$

such that for any $\theta \in \Theta_{0}^{\prime}$,

$$
\left|\theta y_{0}^{b}+u_{0}\right| \geq \frac{1}{4}\left|\Theta_{0}\right| \cdot\left|y_{0}\right|^{b} .
$$

where, "=" can be reached when $u_{0}$ is set to be minus the middle point of the interval $\left[\underline{\theta} y_{0}^{b}, \bar{\theta} y_{0}^{b}\right]$.

Hence, by (20)-(21), it is obvious that for any $\theta \in \Theta_{0}^{\prime}$ and any $w_{1} \in[-w, w]$,

$$
\left|y_{1}\right|=\left|\theta y_{0}^{b}+u_{0}+w_{1}\right| \geq\left|\theta y_{0}^{b}+u_{0}\right|-w \geq \frac{1}{8}\left|\Theta_{0}\right| \cdot\left|y_{0}\right|^{b} \geq \frac{8}{w}\left|y_{0}\right|^{\sqrt{b}}
$$

${ }^{1}$ This choice can only be a causal function of the observed data so far, i.e., $\left\{y_{0}\right\}$. It cannot depend on the unknown parameter $\theta$. 
Then by (22) and (26), we have

$$
\frac{\left|y_{1}\right|^{b}}{\left|y_{0}\right|^{b}} \geq\left(\frac{8}{w}\right)^{b}\left|y_{0}\right|^{b \sqrt{b}-b} \geq 8
$$

which implies that $\left|y_{1}\right|>\left|y_{0}\right|$.

Let us consider the case $\theta \in \Theta_{0}^{\prime}$. At the time $t=1$, with a new observation $y_{1}$ which satisfies the inequality (26), the uncertainty of $\theta$ can be further reduced as follows: Since $\theta$ satisfies the equation (24), i.e.,

$$
\theta=\frac{y_{1}-u_{0}}{y_{0}^{b}}-\frac{w_{1}}{y_{0}^{b}}
$$

with the data $\left\{y_{1}, y_{0}, u_{0}\right\}$ available and with no more knowledge about $w_{1}$ than $\left|w_{1}\right| \leq w$, a natural estimate of $\theta$ would be

$$
\hat{\theta}_{1}:=\frac{y_{1}-u_{0}}{y_{0}^{b}}
$$

Here, we only consider the case where $\hat{\theta}_{1}$ falls in the interval $\Theta_{0}^{\prime}$. This corresponds to some possible values of $y_{1}$.

Then by $(28)$, due to that $w_{1}$ can be any value on the interval $[-w, w], \hat{\theta}_{1}$ can be any value on the interval

$$
\Theta_{1}:=\left[\theta-\frac{w}{\left|y_{0}\right|^{b}}, \quad \theta+\frac{w}{\left|y_{0}\right|^{b}}\right] \cap \Theta_{0}^{\prime} .
$$

Since by (20) and (25)

$$
\left|\Theta_{0}^{\prime}\right| \geq \frac{2 w}{\left|y_{0}\right|^{b}}
$$

it is easy to check that

$$
\left|\Theta_{1}\right| \geq \frac{w}{\left|y_{0}\right|^{b}}
$$

The proceeding analysis exhibits the following fact: For any $\theta \in \Theta_{1}$, there always exists some $w_{1} \in[-w, w]$ such that the output at the time $t=1$ is the $y_{1}$ observed. Therefore, any $\theta \in \Theta_{1}$ is possible with no contradiction to the data $\left\{y_{1}, y_{0}, u_{0}\right\} .^{2}$

\footnotetext{
${ }^{2}$ In other words, based on the observed data $\left\{y_{1}, y_{0}, u_{0}\right\}$, the uncertainty interval of $\theta$ can at most be reduced from $\Theta_{0}$ to $\Theta_{1}$.
} 
Then, with $\Theta_{1}$ as the uncertainty interval of $\theta$ at the time $t=1$, again by the system dynamics

$$
y_{2}=\theta y_{1}^{b}+u_{1}+w_{2}
$$

for any fixed choice ${ }^{3}$ of $u_{1} \in \mathbb{R}^{1}$, there always exists some interval

$$
\Theta_{1}^{\prime} \subset \Theta_{1}
$$

with the length

$$
\left|\Theta_{1}^{\prime}\right| \geq \frac{1}{4}\left|\Theta_{1}\right|
$$

such that for any $\theta \in \Theta_{1}^{\prime}$,

$$
\left|\theta y_{1}^{b}+u_{1}\right| \geq \frac{1}{4}\left|\Theta_{1}\right| \cdot\left|y_{1}\right|^{b} \geq \frac{w}{4} \frac{\left|y_{1}\right|^{b}}{\left|y_{0}\right|^{b}}
$$

where the last inequality follows from (29). Then we have for any $\theta \in \Theta_{1}^{\prime}$ and any $w_{2} \in$ $[-w, w]$

$$
\begin{aligned}
\left|y_{2}\right| & =\left|\theta y_{1}^{b}+u_{1}+w_{2}\right| \geq \frac{w}{4} \frac{\left|y_{1}\right|^{b}}{\left|y_{0}\right|^{b}}-w \geq \frac{w}{8} \frac{\left|y_{1}\right|^{b}}{\left|y_{0}\right|^{b}} \\
& \geq\left(\frac{8}{w}\right)^{\sqrt{b}-1}\left|y_{1}\right|^{b-\sqrt{b}}=\left(\frac{8}{w}\right)^{\sqrt{b}-2}\left|y_{1}\right|^{b-2 \sqrt{b}} \cdot \frac{8}{w}\left|y_{1}\right|^{\sqrt{b}} \\
& \geq \frac{8}{w}\left|y_{1}\right|^{\sqrt{b}}
\end{aligned}
$$

where the first inequality follows from (32), the second inequality follows from (27), the third inequality follows from (26), and the fourth inequality follows from (23) and $\left|y_{1}\right|>$ $\left|y_{0}\right|$. And furthermore, it is obvious by (33) and (22) that

$$
\frac{\left|y_{2}\right|^{b}}{\left|y_{1}\right|^{b}} \geq\left(\frac{8}{w}\right)^{b}\left|y_{1}\right|^{b \sqrt{b}-b}>\left(\frac{8}{w}\right)^{b}\left|y_{0}\right|^{b \sqrt{b}-b} \geq 8,
$$

which implies that $\left|y_{2}\right|>\left|y_{1}\right|$.

\footnotetext{
${ }^{3}$ This choice can only be a causal function of the observed data so far, i.e., $\left\{y_{0}, y_{1}, u_{0}\right\}$. It cannot depend on the unknown parameter $\theta$.
} 
Let us consider the case $\theta \in \Theta_{1}^{\prime}$. First, note that by (27), (29) and (31)

$$
\left|\Theta_{1}^{\prime}\right| \geq \frac{1}{4}\left|\Theta_{1}\right| \geq \frac{w}{4\left|y_{0}\right|^{b}} \geq \frac{2 w}{\left|y_{1}\right|^{b}} .
$$

At the time $t=2$, similarly to the time $t=1$ analyzed before, there exists some interval $\Theta_{2} \subset \Theta_{1}^{\prime}$ with $\left|\Theta_{2}\right| \geq \frac{w}{\left|y_{1}\right|^{b}}$ such that for any $\theta \in \Theta_{2}$, there always exists some $w_{2} \in[-w, w]$ such that the output at the time $t=2$ is the $y_{2}$ observed. Therefore, any $\theta \in \Theta_{2}$ is possible with no contradiction to the data $\left\{y_{2}, y_{1}, y_{0}, u_{1}, u_{0}\right\}$. Then, with $\Theta_{2}$ as the uncertainty interval of $\theta$ at the time $t=2$, by the system dynamics

$$
y_{3}=\theta y_{2}^{b}+u_{2}+w_{3}
$$

no matter what was the choice of $u_{2}$, there always exists some interval

$$
\Theta_{2}^{\prime} \subset \Theta_{2}
$$

with the length

$$
\left|\Theta_{2}^{\prime}\right| \geq \frac{1}{4}\left|\Theta_{2}\right|
$$

such that for any $\theta \in \Theta_{2}^{\prime}$,

$$
\left|\theta y_{2}^{b}+u_{2}\right| \geq \frac{1}{4}\left|\Theta_{2}\right| \cdot\left|y_{2}\right|^{b} \geq \frac{w}{4} \frac{\left|y_{2}\right|^{b}}{\left|y_{1}\right|^{b}}
$$

Then by (34) and (35), it is obvious that for any $\theta \in \Theta_{2}^{\prime}$ and any $w_{3} \in[-w, w]$,

$$
\begin{aligned}
\left|y_{3}\right| & =\left|\theta y_{2}^{b}+u_{2}+w_{3}\right| \geq \frac{w}{4} \frac{\left|y_{2}\right|^{b}}{\left|y_{1}\right|^{b}}-w \geq \frac{w}{8} \frac{\left|y_{2}\right|^{b}}{\left|y_{1}\right|^{b}} \\
& \geq\left(\frac{8}{w}\right)^{\sqrt{b}-1}\left|y_{2}\right|^{b-\sqrt{b}}=\left(\frac{8}{w}\right)^{\sqrt{b}-2}\left|y_{2}\right|^{b-2 \sqrt{b}} \cdot \frac{8}{w}\left|y_{2}\right|^{\sqrt{b}} \\
& \geq \frac{8}{w}\left|y_{2}\right|^{\sqrt{b}}
\end{aligned}
$$

where the last two inequalities follow from (33), (23) and that $\left|y_{2}\right|>\left|y_{1}\right|>\left|y_{0}\right|$. And furthermore, it is obvious by (37) and (22) that

$$
\frac{\left|y_{3}\right|^{b}}{\left|y_{2}\right|^{b}} \geq\left(\frac{8}{w}\right)^{b}\left|y_{2}\right|^{b \sqrt{b}-b}>\left(\frac{8}{w}\right)^{b}\left|y_{0}\right|^{b \sqrt{b}-b} \geq 8,
$$


which implies that $\left|y_{3}\right|>\left|y_{2}\right|$.

This process can be continued infinitely for the times $t=3,4, \ldots$ and we can similarly construct the intervals

$$
\Theta_{t}^{\prime} \subset \Theta_{t} \subset \Theta_{t-1}^{\prime} \quad \text { with }\left|\Theta_{t}\right| \geq \frac{w}{\left|y_{t-1}\right|^{b}} \text { and }\left|\Theta_{t}^{\prime}\right| \geq \frac{1}{4}\left|\Theta_{t}\right|
$$

such that any $\theta \in \Theta_{t}$ is possible with no contradiction to the data $\left\{y_{t}, \ldots, y_{0}, u_{t-1}, \ldots, u_{0}\right\}$, and for any $\theta \in \Theta_{t}^{\prime}$ and any $w_{t+1} \in[-w, w]$,

$$
\left|y_{t+1}\right| \geq \frac{8}{w}\left|y_{t}\right|^{\sqrt{b}} \text { and } \frac{\left|y_{t+1}\right|^{b}}{\left|y_{t}\right|^{b}} \geq 8 .
$$

Finally, $\Theta_{\infty}:=\lim _{t \rightarrow \infty} \Theta_{t}^{\prime} \neq \emptyset$ and it happens to any $\theta \in \Theta_{\infty}$ with no contradiction to the data $\left\{y_{0}, y_{1}, \ldots, u_{0}, u_{1}, \ldots\right\}$ (or in other words, a suitable sequence of $\left\{w_{t}\right\}$ can be found) that

$$
\left|y_{t}\right| \nearrow \infty
$$

Proof of Theorem 2.2: Let $u_{0}=0$. Starting from $t=1$, let $u_{t}=0$ if $f\left(y_{t-1}\right)=0$. Let $t_{0} \geq 1$ be the first time such that $f\left(y_{t_{0}-1}\right) \neq 0$. (If $f\left(y_{t-1}\right)=0$ for all $t \geq 1$, then it is easy to check by (5) that $\sup _{t \geq 0}\left|y_{t}\right| \leq w$. Hence we only need to consider the case where $t_{0}<\infty$.) Then for $t \geq t_{0}$, we do parameter estimation and design the corresponding certainty equivalence controller as follows.

The parameter estimation method is similar to that in the proof of Theorem 2.1. The only difference is that here $f\left(y_{i}\right)$ is in place of $y_{i}^{b}$.

For any $t \geq t_{0}$, let

$$
i_{t}:=\underset{0 \leq i \leq t-1}{\operatorname{argmax}}\left|f\left(y_{i}\right)\right| .
$$

The parameter estimate at the time $t \geq t_{0}$ is chosen to be

$$
\hat{\theta}_{t}:=\frac{y_{i_{t}+1}-u_{i_{t}}}{f\left(y_{i_{t}}\right)} .
$$


The control sequence is defined as

$$
u_{t}=-\hat{\theta}_{t} f\left(y_{t}\right), \quad \text { for } t \geq t_{0} .
$$

Then for $t \geq t_{0}$, the closed-loop dynamics is

$$
y_{t+1}=\frac{-w_{i_{t}+1}}{f\left(y_{i_{t}}\right)} f\left(y_{t}\right)+w_{t+1} .
$$

Therefore, noting that the noise are uniformly bounded as (2), we have

$$
\left|y_{t+1}\right| \leq \frac{w}{\left|f\left(y_{i_{t}}\right)\right|}\left|f\left(y_{t}\right)\right|+w, \quad \text { for any } t \geq t_{0}
$$

Then by (39), we have

$$
\left|y_{t+1}\right| \leq \frac{w}{\max _{0 \leq i \leq t-1}\left|f\left(y_{i}\right)\right|}\left|f\left(y_{t}\right)\right|+w, \quad \text { for any } t \geq t_{0} .
$$

Now we use a contradiction argument to prove that the outputs are uniformly bounded as (4). Suppose on the contrary, there exist some $y_{0} \in \mathbb{R}^{1}$, and some $\theta$ and a sequence of $\left\{w_{t}\right\}$ satisfying the assumptions A1)-A2) such that for the feedback control law proposed above,

$$
\sup _{t \geq 0}\left|y_{t}\right|=\infty
$$

Then by $t_{0}<\infty$, we have

$$
\sup _{t \geq t_{0}}\left|y_{t}\right|=\infty
$$

If for this sequence $\left\{y_{t}, t \geq t_{0}\right\}, \sup _{t \geq t_{0}}\left|f\left(y_{t}\right)\right| \leq F<\infty$, then by (40) and the definition of $t_{0}$, it is easy to check that for any $t \geq t_{0}$,

$$
\left|y_{t+1}\right| \leq \frac{w F}{\left|f\left(y_{t_{0}-1}\right)\right|}+w<\infty
$$

So we only need to consider the case where $\sup _{t \geq t_{0}}\left|f\left(y_{t}\right)\right|=\infty$. From this sequence $\left\{\left|f\left(y_{t}\right)\right|, t \geq t_{0}\right\}$, we can pick out a subsequence $\left\{\left|f\left(y_{t_{k}}\right)\right|, k \geq 1\right\}$ which monotonously increasing to the infinity and also for any $k=1,2, \ldots$

$$
\left|f\left(y_{t}\right)\right| \leq\left|f\left(y_{t_{k}}\right)\right|<\left|f\left(y_{t_{k+1}}\right)\right| \quad \text { for any } t_{k}<t<t_{k+1},
$$


and $\left|f\left(y_{t_{1}}\right)\right|>\sup _{|x| \leq M_{1}}|f(x)|$, which by (7) means that

$$
\left|y_{t_{k}}\right|>M_{1}, \quad k=1,2, \ldots
$$

Now, for any $k=2,3, \ldots$, by (40), we have

$$
\left|y_{t_{k+1}}\right| \leq \frac{w}{\max _{0 \leq i \leq t_{k+1}-2}\left|f\left(y_{i}\right)\right|}\left|f\left(y_{t_{k+1}-1}\right)\right|+w .
$$

By (41), it is easy to check that

$$
\left|f\left(y_{t_{k+1}-1}\right)\right| \leq\left|f\left(y_{t_{k}}\right)\right| \text { and } \max _{0 \leq i \leq t_{k+1}-2}\left|f\left(y_{i}\right)\right| \geq\left|f\left(y_{t_{k-1}}\right)\right|
$$

Hence, we have

$$
\left|y_{t_{k+1}}\right| \leq \frac{w}{\left|f\left(y_{t_{k-1}}\right)\right|}\left|f\left(y_{t_{k}}\right)\right|+w .
$$

Take logarithm on both sides of (55), and noting that $\left|f\left(y_{t_{k}}\right)\right|>\left|f\left(y_{t_{k-1}}\right)\right|$, we have

$$
\begin{aligned}
\log \left|y_{t_{k+1}}\right| & \leq \log \left(\frac{w}{\left|f\left(y_{t_{k-1}}\right)\right|}\left|f\left(y_{t_{k}}\right)\right|+w\right) \\
& <\log \left(2 w \frac{\left|f\left(y_{t_{k}}\right)\right|}{\left|f\left(y_{t_{k-1}}\right)\right|}\right) \\
& =\log \left|f\left(y_{t_{k}}\right)\right|-\log \left|f\left(y_{t_{k-1}}\right)\right|+\log (2 w) .
\end{aligned}
$$

Then by the assumption A3) and (42), we have

$$
\log \left|f\left(y_{t_{k+1}}\right)\right| \leq b\left(\log \left|f\left(y_{t_{k}}\right)\right|-\log \left|f\left(y_{t_{k-1}}\right)\right|\right)+\log \left((2 w)^{b} a_{1}\right)
$$

Then it follows by Lemma 3.5 in $[6]$ that for $b<4,\left\{\log \left|f\left(y_{t_{k}}\right)\right|\right\}$ cannot be monotonously increasing to the infinity, which contradicts to the definition of $\left\{\left|f\left(y_{t_{k}}\right)\right|\right\}$. This concludes the contradiction argument.

Proof of Theorem 2.3: We show that if $b \geq 4$ and $\left|y_{0}\right|$ is sufficiently large, then for any feedback control law, there always exist some $\theta$ and $\left\{w_{t}\right\}$ satisfying the assumptions A1)-A2) such that the absolute values of the outputs monotonously increase to the infinity:

$$
\left|y_{t}\right| \nearrow \infty
$$


The proof is similar to that of Theorem 2.1. Hence, we only mention the differences here. Let

$$
f^{\prime}(x):=a_{2}^{\frac{1}{b-1}} f(x) \quad \text { and } \quad w^{\prime}:=a_{2}^{\frac{1}{b-1}} w .
$$

We consider sufficiently large $\left|y_{0}\right|$ satisfying the following inequalities:

$$
\begin{gathered}
\frac{1}{4}\left|\Theta_{0}\right| \cdot\left|f\left(y_{0}\right)\right| \geq \max \left\{2 w, 2 M_{2}\right\} \\
\frac{1}{8}\left|\Theta_{0}\right| \cdot\left|f^{\prime}\left(y_{0}\right)\right|^{\frac{b-\sqrt{b}}{b}} \geq \frac{8}{w^{\prime}} \\
\left(\frac{8}{w^{\prime}}\right)^{b}\left|f^{\prime}\left(y_{0}\right)\right|^{\sqrt{b}-1} \geq \max \left\{8, \frac{8 M_{2}}{w}\right\} \\
\left(\frac{8}{w^{\prime}}\right)^{b(\sqrt{b}-2)}\left|f^{\prime}\left(y_{0}\right)\right|^{b-2 \sqrt{b}} \geq 1 .
\end{gathered}
$$

At the time $t=0$, the system dynamics evolves as

$$
y_{1}=\theta f\left(y_{0}\right)+u_{0}+w_{1}
$$

Similarly, for any $u_{0}$, there exists $\Theta_{0}^{\prime} \subset \Theta_{0}$ with $\left|\Theta_{0}^{\prime}\right| \geq \frac{1}{4}\left|\Theta_{0}\right|$ such that for any $\theta \in \Theta_{0}^{\prime}$,

$$
\left|\theta f\left(y_{0}\right)+u_{0}\right| \geq \frac{1}{4}\left|\Theta_{0}\right| \cdot\left|f\left(y_{0}\right)\right| \text {. }
$$

Hence by (46) it is obvious that for any $\theta \in \Theta_{0}^{\prime}$ and any $w_{1} \in[-w, w]$,

$$
\left|y_{1}\right|=\left|\theta f\left(y_{0}\right)+u_{0}+w_{1}\right| \geq\left|\theta f\left(y_{0}\right)+u_{0}\right|-w \geq \frac{1}{8}\left|\Theta_{0}\right| \cdot\left|f\left(y_{0}\right)\right| \geq M_{2} .
$$

Then by (8)

$$
\left|f\left(y_{1}\right)\right| \geq a_{2}\left(\frac{1}{8}\left|\Theta_{0}\right|\right)^{b} \cdot\left|f\left(y_{0}\right)\right|^{b}
$$

Hence by (45) and (47)

$$
\left|f^{\prime}\left(y_{1}\right)\right| \geq\left(\frac{1}{8}\left|\Theta_{0}\right|\right)^{b}\left|f^{\prime}\left(y_{0}\right)\right|^{b} \geq\left(\frac{8}{w^{\prime}}\right)^{b}\left|f^{\prime}\left(y_{0}\right)\right|^{\sqrt{b}} .
$$

Then by (53) and (48), we have

$$
\frac{\left|f\left(y_{1}\right)\right|}{\left|f\left(y_{0}\right)\right|}=\frac{\left|f^{\prime}\left(y_{1}\right)\right|}{\left|f^{\prime}\left(y_{0}\right)\right|} \geq\left(\frac{8}{w^{\prime}}\right)^{b}\left|f^{\prime}\left(y_{0}\right)\right|^{\sqrt{b}-1} \geq \max \left\{8, \frac{8 M_{2}}{w}\right\} .
$$


Next, the generation of the interval $\Theta_{1} \subset \Theta_{0}^{\prime}$ is similar to the process in the proof of Theorem 2.1, only with $y_{0}^{b}$ replaced by $f\left(y_{0}\right)$ :

$$
\Theta_{1}:=\left[\theta-\frac{w}{\left|f\left(y_{0}\right)\right|}, \quad \theta+\frac{w}{\left|f\left(y_{0}\right)\right|}\right] \cap \Theta_{0}^{\prime} .
$$

And we also have

$$
\left|\Theta_{1}\right| \geq \frac{w}{\left|f\left(y_{0}\right)\right|}
$$

Then by the following equation

$$
y_{2}=\theta f\left(y_{1}\right)+u_{1}+w_{2},
$$

for any $u_{1}$, there always exists some interval $\Theta_{1}^{\prime} \subset \Theta_{1}$ with $\left|\Theta_{1}^{\prime}\right| \geq \frac{1}{4}\left|\Theta_{1}\right|$ such that for any $\theta \in \Theta_{1}^{\prime}$

$$
\left|\theta f\left(y_{1}\right)+u_{1}\right| \geq \frac{1}{4}\left|\Theta_{1}\right| \cdot\left|f\left(y_{1}\right)\right| \geq \frac{w}{4} \frac{\left|f\left(y_{1}\right)\right|}{\left|f\left(y_{0}\right)\right|}
$$

where the last inequality follows from (55). Then by (54), it is obvious that for any $\theta \in \Theta_{1}^{\prime}$ and any $w_{2} \in[-w, w]$,

$$
\begin{aligned}
\left|y_{2}\right| & =\left|\theta f\left(y_{1}\right)+u_{1}+w_{2}\right| \geq \frac{w}{4} \frac{\left|f\left(y_{1}\right)\right|}{\left|f\left(y_{0}\right)\right|}-w \geq \frac{w}{8} \frac{\left|f\left(y_{1}\right)\right|}{\left|f\left(y_{0}\right)\right|} \\
& \geq \frac{w}{8}\left(\frac{8}{w^{\prime}}\right)^{b}\left|f^{\prime}\left(y_{0}\right)\right|^{\sqrt{b}-1} \\
& \geq M_{2} .
\end{aligned}
$$

Then by (8), (45), (53) and (49)

$$
\begin{aligned}
\left|f^{\prime}\left(y_{2}\right)\right| & =a_{2}^{\frac{1}{b-1}}\left|f\left(y_{2}\right)\right| \geq a_{2}^{\frac{b}{b-1}}\left|y_{2}\right|^{b} \\
& \geq a_{2}^{\frac{b}{b-1}}\left(\frac{w}{8} \cdot \frac{\left|f\left(y_{1}\right)\right|}{\left|f\left(y_{0}\right)\right|}\right)^{b}=\left(\frac{w^{\prime}}{8} \cdot \frac{\left|f^{\prime}\left(y_{1}\right)\right|}{\left|f^{\prime}\left(y_{0}\right)\right|}\right)^{b} \\
& \geq\left(\frac{8}{w^{\prime}}\right)^{b(\sqrt{b}-1)}\left|f^{\prime}\left(y_{1}\right)\right|^{b-\sqrt{b}} \\
& =\left(\frac{8}{w^{\prime}}\right)^{b(\sqrt{b}-2)}\left|f^{\prime}\left(y_{1}\right)\right|^{b-2 \sqrt{b}} \cdot\left(\frac{8}{w^{\prime}}\right)^{b}\left|f^{\prime}\left(y_{1}\right)\right|^{\sqrt{b}} \\
& \geq\left(\frac{8}{w^{\prime}}\right)^{b}\left|f^{\prime}\left(y_{1}\right)\right|^{\sqrt{b}} .
\end{aligned}
$$


And furthermore, it is obvious by (48) that

$$
\frac{\left|f^{\prime}\left(y_{2}\right)\right|}{\left|f^{\prime}\left(y_{1}\right)\right|} \geq\left(\frac{8}{w^{\prime}}\right)^{b}\left|f^{\prime}\left(y_{1}\right)\right|^{\sqrt{b}-1}>\left(\frac{8}{w^{\prime}}\right)^{b}\left|f^{\prime}\left(y_{0}\right)\right|^{\sqrt{b}-1} \geq \max \left\{8, \frac{8 M_{2}}{w}\right\} .
$$

This process can be continued infinitely for the times $t=2,3, \ldots$ and we can similarly construct the intervals

$$
\Theta_{t}^{\prime} \subset \Theta_{t} \subset \Theta_{t-1}^{\prime} \quad \text { with }\left|\Theta_{t}\right| \geq \frac{w}{\left|f\left(y_{t-1}\right)\right|} \text { and }\left|\Theta_{t}^{\prime}\right| \geq \frac{1}{4}\left|\Theta_{t}\right|
$$

such that any $\theta \in \Theta_{t}$ is possible with no contradiction to the data $\left\{y_{t}, \ldots, y_{0}, u_{t-1}, \ldots, u_{0}\right\}$, and for any $\theta \in \Theta_{t}^{\prime}$ and any $w_{t+1} \in[-w, w]$,

$$
\begin{aligned}
& \qquad\left|f^{\prime}\left(y_{t+1}\right)\right| \geq\left(\frac{8}{w^{\prime}}\right)^{b}\left|f^{\prime}\left(y_{t}\right)\right|^{\sqrt{b}}, \frac{\left|f^{\prime}\left(y_{t+1}\right)\right|}{\left|f^{\prime}\left(y_{t}\right)\right|} \geq \max \left\{8, \frac{8 M_{2}}{w}\right\} \\
& \text { and } \quad\left|y_{t+1}\right| \geq \frac{w}{8}\left(\frac{8}{w^{\prime}}\right)^{b}\left|f^{\prime}\left(y_{t-1}\right)\right|^{\sqrt{b}-1} \text {. }
\end{aligned}
$$

Finally, $\Theta_{\infty}:=\lim _{t \rightarrow \infty} \Theta_{t}^{\prime} \neq \emptyset$ and it happens to any $\theta \in \Theta_{\infty}$ with no contradiction to the data $\left\{y_{0}, y_{1}, \ldots, u_{0}, u_{1}, \ldots\right\}$ (or in other words, a suitable sequence of $\left\{w_{t}\right\}$ can be found) that

$$
\left|f^{\prime}\left(y_{t}\right)\right| \nearrow \infty
$$

which by (58) implies

$$
\sup _{t \geq 0}\left|y_{t}\right|=\infty
$$

\section{References}

[1] L. Guo. On critical stability of discrete-time adaptive nonlinear control. IEEE Trans. Autom. Contr., 42:1488-1499, 1997.

[2] L. Guo and C. Wei. LS-based discrete-time adaptive nonlinear control: Feasability and limitations. Science in China, Series E, 39(3):255-269, 1996. 
[3] I. Kanellakopoulos. A discrete-time adaptive nonlinear system. IEEE Trans. Autom. Contr., 39:2362-2364, 1994.

[4] L.-L. Xie and L. Guo. Fundamental limitations of discrete-time adaptive nonlinear control. IEEE Trans. Autom. Contr., 44:1777-1782, 1999.

[5] L.-L. Xie and L. Guo. Adaptive control of discrete-time nonlinear systems with structural uncertainties. In Lectures on Systems, Control, and Information, pages 49-90. AMS/IP, 2000.

[6] L.-L. Xie and L. Guo. How much uncertainty can be dealt with by feedback? IEEE Trans. Autom. Contr., 45:2203-2217, 2000.

[7] J. Zhao and I. Kanellakopoulos. Active identification for discrete-time nonlinear control-Part I: output-feedback systems. IEEE Trans. Autom. Contr., 47:210-224, 2002. 